\title{
Dynamic Detonation Stabilization in Supersonic Expanding Channels
}

\author{
Xiaodong Cai ${ }^{1}$, Ralf Deiterding ${ }^{2}$, Jianhan Liang ${ }^{1 *}$, Dezun Dong ${ }^{3}$, Mingbo Sun ${ }^{1}$
}

${ }^{1}$ Science and Technology on Scramjet Laboratory,

National University of Defense Technology, Changsha, 410073, China

${ }^{2}$ Aerodynamics and Flight Mechanics Research Group, University of Southampton,

Highfield Campus, Southampton SO17 1BJ, United Kingdom

${ }^{3}$ College of Computer, National University of Defense Technology,

Changsha, 410073, China

*Corresponding author: jhleon@vip.sina.com 


\section{Abstract:}

In the present work, dynamic detonation stabilization in expanding channels is numerically investigated by injecting a hot jet into a hydrogen-oxygen combustible mixture flowing at supersonic speed. The two-dimensional reactive Navier-Stokes equations and one-step two-species reaction model are solved using a hybrid sixth-order WENO-Centered Difference (CD) scheme based on the SAMR (Structured Adaptive Mesh Refinement) framework. The results show that the highly unstable shear layer interactions with the unburned jet resulting from the Prandtl-Meyer expansion fan result in numerous large-scale vortices, which contribute significantly to rapid turbulent mixing and diffusion effects. This can further facilitate the consumption of the unburned jet and its subsequent heat release to support the dynamically stationary propagation of detonation. Meanwhile, detonation attenuation in the supersonic flow can be also effectively suppressed because of the formation of a hydrodynamic channel associated with a corresponding hydrodynamic throat. It is indicated that the shear layer interactions with the unburned jet and the generation of hydrodynamic channel can both play important roles in dynamically stationary propagation of detonation in supersonic expanding channels after the shutdown of the hot jet. With the increase of the expansion angle, the enlarged unburned jet is gradually extended out of the sonic line, and the deficit of heat release cannot contribute to stationary propagation of detonation, thus eventually leading to detonation failure. It is indicated that there might exist a critical angle $\theta_{\mathrm{CT}}$. Dynamic stabilization of detonation can be realized in expanding channels when the angle is smaller than $\theta_{\mathrm{CT}}$, while the detonation propagates below the CJ velocity and finally fails when the angle is larger than $\theta_{\mathrm{CT}}$. Through the control of a moving boundary by dynamically changing the expansion angle, the continuous detonation attenuation can be effectively suppressed and finally turned to forward propagation successfully, indicating that dynamically stationary propagation of detonation can be 
realized through the dynamic control of a moving boundary.

Key words: Supersonic expanding channel; Stationary detonation propagation; Stabilization mechanism; Dynamic control

\section{Introduction}

Scramjets have become one of the first choices for hypersonic air-breathing propulsion systems thanks to the superior performance at high Mach numbers $(\mathrm{Ma} \geq 5.0)$. Due to the inherent theoretical advantage over deflagration combustion [1-2], it is believed that the performance of the scramjet might be improved significantly if continuous detonation-driven combustion can be realized effectively in supersonic combustible mixtures of scramjet combustors.

Based on the idea of a detonation-driven scramjet (DDS), a series of numerical and experimental investigations have been conducted on detonation combustion in supersonic combustible mixtures of both simplified straight channels [3-5], which mainly concentrate on detonation initiation and its propagation characteristics in supersonic combustible mixtures, and cavity-based straight channels [6] focusing on detonation stabilization owing to cavity oscillations [7-9].

Taking into account that the actual combustors are usually expanding for thrust propulsion, recently two-dimensional detonation simulations in supersonic expanding channels have been carried out [10]. It is speculated that the detonation front can be maintained almost in the same position in the expanding channel for the given expanding angle after the shutdown of the hot jet, suggesting the formation of dynamically quasi-steady propagation of detonation. Different from straight channels, the detonation product in expanding channels is expected to be able to have more free expansion due to the Prandtl-Meyer expansion fan. This can theoretically result in further attenuation of Chapman- 
Jouget $(\mathrm{CJ})$ detonation and even detonation failure. Compared with straight channels, rather than further attenuation or detonation failure, dynamic detonation stabilization is supposed to be realized in the expanding channel after the shutdown of the hot jet; however, the intrinsic mechanism is not understood. It is surmised that periodic formation and rapid consumption of the unburned jet resulting from the Prandtl-Meyer expansion fan can contribute to dynamically stationary propagation of detonation. The formation and consumption of the unburned mixture behind the detonation front are highly dependent on diffusion and mixing effects [11-13], which are speculated to be significantly subjected to the interactions between highly unstable shear layers and the unburned jet.

$\mathrm{Lu}$ and Braun [14] have pointed out that it is an important problem for detonation-driven engines in practice to be able to sustain a detonation wave for a long duration and control it in some fashion when bringing the concept to reality. Detonation is a supersonic shock wave coupled with reaction zone that propagates normally at an average CJ velocity. Therefore, it is very critical to dynamically stabilize the detonation in supersonic combustors with limited length when it propagates at the CJ state. For the DDS in expanding channels, first of all it is vital to reveal the mechanism of dynamically stationary propagation of detonation after the shutdown of the hot jet, and then to establish which possible control method might be reasonably proposed on the basis of the mechanism. In order to clarify the mechanism of stationary propagation of detonation in supersonic expanding channels, in the present work the reactive Navier-Stokes (NS) equations with a simple reaction model [15] are solved using a hybrid six-order WENO-CD (Weighted Essentially NonOscillatory-Centered Difference) scheme [16-17] utilizing the open-source program AMROC [18-21] (Adaptive Mesh Refinement Object-oriented $\mathrm{C}++$ ) based on an SAMR (Structured Adaptive Mesh Refinement) framework. It is reported that in inviscid detonation simulations the absence of smallscale turbulent interactions, normally not properly accounted for, leads to significantly lower burning 
rates than observed experimentally and the associated diffusive effects can control the reaction of approximately half of the gases passing across the detonation front in unstable cellular detonations [22-23]. Therefore, in the present work the utilization of the NS equations combined with the lowdissipation hybrid high-order scheme is essential to ensure the proper resolution of these small-scale turbulent interactions associated with diffusion and mixing effects for more accurate physical descriptions of detonation combustion in supersonic expanding channels when the unburned jet is generated behind the detonation front. This work is part of ongoing research, aimed at providing information to improve the overall understanding of detonation stabilization in supersonic combustible mixtures.

The remainder of this paper is organized as follows: Section 2 introduces the computational model, including governing equations, numerical methods and computational setup. The stationary propagation of detonation is presented in Section 3, where the dynamically stationary propagation, stabilization mechanism and subsequent control application are further investigated. Finally, Section 4 concludes the paper.

\section{Computational model}

\subsection{Governing equations}

The present work utilizes the NS equations with the one-step two-species (reactant and product) chemistry model as governing equations. The two-dimensional governing equations are expressed as follows:

$$
\frac{\partial U}{\partial t}+\frac{\partial\left(F_{c o n v}-F_{\text {diff }}\right)}{\partial x}+\frac{\partial\left(H_{c o n v}-H_{\text {diff }}\right)}{\partial y}=S_{\text {chem }},
$$

where the vector of state is 


$$
U=\left(\rho, \rho u, \rho v, \rho e, \rho Y_{1}\right)
$$

where $\rho, u, v, e$ and $Y_{1}$ are the total density, the velocity in the $x$-direction, the velocity in the $y$-direction, the total energy per unit mass and the mass fraction of the reactant, respectively.

The convective and diffusive fluxes are

$$
\begin{gathered}
F_{c o n v}=\left(\rho u, \rho u^{2}+p, \rho u v, \rho u e+u p, \rho u Y_{1}\right), \\
H_{c o n v}=\left(\rho v, \rho u v, \rho v^{2}+p, \rho v e+v p, \rho v Y_{1}\right) \\
F_{d i f f}=\left(0, \tau_{x x}, \tau_{x y}, u \tau_{x x}+v \tau_{x y}+k \frac{\partial T}{\partial x}+\rho h_{1} D_{1} \frac{\partial Y_{1}}{\partial x}+\rho h_{2} D_{2} \frac{\partial Y_{2}}{\partial x}, \rho D_{1} \frac{\partial Y_{1}}{\partial x}\right), \\
H_{d i f f}=\left(0, \tau_{y x}, \tau_{y y}, u \tau_{y x}+v \tau_{y y}+k \frac{\partial T}{\partial y}+\rho h_{1} D_{1} \frac{\partial Y_{1}}{\partial y}+\rho h_{2} D_{2} \frac{\partial Y_{2}}{\partial x}, \rho D_{1} \frac{\partial Y_{1}}{\partial y}\right),
\end{gathered}
$$

and the reactive source term is

$$
S_{\text {chem }}=\left(0,0,0,0,0, \dot{w}_{1}\right) .
$$

Here, $\tau$ is the stress tensor; $h_{1}$ and $h_{2}$ are the enthalpies of the reactant and product, respectively; $\dot{w}_{1}$ is the mass production rate of the reactant. $\mu, k, D_{1}, D_{2}$ are the mixture viscosity, the thermal conductivity, and the mass diffusivities of the reactant and the product, respectively. $e$ is defined as

$$
e=\frac{p}{\rho(\gamma-1)}+\frac{\left(u^{2}+v^{2}\right)}{2}+Y_{1} q
$$

where $q$ is the heat release per unit mass. The stresses read

$$
\tau_{x x}=\mu\left(\frac{4}{3} \frac{\partial u}{\partial x}-\frac{2}{3} \frac{\partial v}{\partial y}\right), \tau_{x y}=\tau_{y x}=\mu\left(\frac{\partial v}{\partial x}+\frac{\partial u}{\partial y}\right) \text { and } \tau_{y y}=\mu\left(\frac{4}{3} \frac{\partial v}{\partial y}-\frac{2}{3} \frac{\partial u}{\partial x}\right) .
$$

Currently, it is computationally very expensive to conduct high-resolution multi-dimensional detonation simulations solving the NS equations with detailed chemistry. The Arrhenius law that relates chemical reaction rates to temperature variation is widely used as the simplest mode for detonation simulations [24-27]. Here the reaction model is selected and fitted to the physical 
parameters of a $\mathrm{H}_{2} / \mathrm{O}_{2}$ detonation initially at $T=300 \mathrm{~K}$ and $P=6.67 \mathrm{kPa}$ [20]. This defines a marginally stable detonation with the corresponding average detonation cell of $\lambda=25 \mathrm{~mm}$.

For the two species, the calorically perfect model

$$
\gamma=\gamma_{1}=\gamma_{2}, p=\rho R T, R=R_{1}=R_{2}
$$

is used. The mass fraction production rates are given as follows:

$$
\dot{w}_{1}=-\dot{w}_{2}=\rho Y_{1} A \exp \left(\frac{E_{a}}{R T}\right) .
$$

The thermodynamic parameters are shown in Table 1 and yield a CJ velocity of $V_{C J}=1587.84 \mathrm{~m} / \mathrm{s}$. At the end of the $\mathrm{ZND}$ reaction zone, the temperature is about $2500 \mathrm{~K}$ and the pressure is approximately $101.325 \mathrm{kPa}$, which gives the following transport parameters for the onestep model: $T_{\text {ref }}=2500 \mathrm{~K}, \mu_{\text {ref }}=1.07 \times 10^{-4} \mathrm{~Pa} \cdot \mathrm{s}, k_{\text {ref }}=0.148 \mathrm{~W} /(\mathrm{m} \cdot \mathrm{K}), D_{\text {1ref }}=5.5 \times 10^{-4} \mathrm{~m}^{2} / \mathrm{s}$, $D_{2 r e f}=6.4 \times 10^{-4} \mathrm{~m}^{2} / \mathrm{s}$.

Table 1 Thermodynamic parameters of the mixture.

\begin{tabular}{ccc}
\hline Parameters & Values & Unit \\
\hline$T_{\infty}$ & 300 & $\mathrm{~K}$ \\
$p_{\infty}$ & 6.67 & $\mathrm{kPa}$ \\
$\rho_{\infty}$ & 0.077552 & $\mathrm{~kg} / \mathrm{m}^{3}$ \\
$\gamma$ & 1.29499 & \\
$W$ & 0.029 & $\mathrm{~kg} / \mathrm{mol}$ \\
$q$ & 54000 & $\mathrm{~J} / \mathrm{mol}$ \\
$E_{a}$ & 30000 & $\mathrm{~J} / \mathrm{mol}$ \\
$A$ & $6 \times 10^{5}$ & $\mathrm{~s}^{-1}$ \\
\hline
\end{tabular}

By matching the general trends and values at the end of the ZND reaction zone between the one-step and the detailed reaction model, the viscosity and conductivity are given by the Sutherland model, while the mass diffusion is given by a simple expression which includes the inverse 
dependence on pressure, i.e.,

$$
\begin{gathered}
\frac{u}{u_{\text {ref }}}=\left(\frac{T}{T_{\text {ref }}}\right)^{\frac{5}{2}}, \frac{k}{k_{\text {ref }}}=\left(\frac{T}{T_{\text {ref }}}\right)^{\frac{5}{2}}, \\
\frac{D_{1}}{D_{\text {lref }}}=\left(\frac{T}{T_{\text {ref }}}\right)^{\frac{5}{2}} \frac{p_{a t m}}{p}, \frac{D_{2}}{D_{2 \text { ref }}}=\left(\frac{T}{T_{\text {ref }}}\right)^{\frac{5}{2}} \frac{p_{a t m}}{p} .
\end{gathered}
$$

\subsection{Numerical methods}

Typically shock-capturing methods introduce excessive numerical dissipation, which pollutes the diffusive part of the NS equations. However, the methods which can capture accurately the diffusive terms without numerical dissipation usually lack robustness and stability at discontinuities due to a lack of dissipation. Originally developed for large eddy simulations (LES) of turbulence, the hybrid WENO-CD scheme consists of two components: a finite-difference sixth-order WENO scheme to be used at discontinuities and a conservative sixth-order CD scheme for smooth-solution regions. WENO schemes perform well for first-order hyperbolic problems, but introduce plenty of numerical dissipation for second-order mixed equations with physical diffusion. Meanwhile, no numerical viscosity is introduced for schemes developed with centered stencils, but generally numerical instabilities are produced at discontinuities. Through a switch using a shock-based detection technique [28], the hybrid WENO-CD scheme combines both the advantages of WENO and CD schemes: regions of strong discontinuities are approximated by the WENO scheme, while the $\mathrm{CD}$ scheme is used within regions of smooth flow, thus minimizing numerical dissipation to the extent possible.

Due to the stability properties of explicit integration schemes, the preferred practical methods with the ability of inexpensive time adaptation in SAMR are the Runge-Kutta of third or higher order. 
In the present work, the optimal third-order strong stability preserving (SSP) Runge-Kutta scheme is used associated with time-splitting and the fourth-order accurate semi-implicit GRK4A method [29] for source term integration.

\subsection{Computational setup}

The computations are carried out in two-dimensional expanding channels, as depicted in Fig.1. In order to construct the upper expanding wall, the ghost fluid method (GFM) is adopted in the SAMR framework. The numerical stencil by itself is not modified, which will cause a slight diffusion of the boundary location throughout the method. By refining the embedded boundary typically up to the highest available resolution, this problem will be effectively alleviated [30]. Reflecting boundaries with slip wall conditions are used on the upper and lower walls. In the viscous case, a turbulent boundary layer would develop along the inflow channel wall and its thickness will strongly depend on the channel length. Especially at the start of the expansion wall, the boundary layer will mostly detach, migrate partially into the channel interior and will only reestablish at the end of the expansion section. This is rather complicated and can only accurately be described by simulating the entire combustion chamber. In order to eliminate this complexity and to be able to focus the present study just on the dynamically stable propagation of detonation and the subsequent stabilization control in the supersonic expanding channel, the viscous model with slip boundary conditions is opted for not having to deal with boundary layers produced otherwise in the inflow section. Within the lower wall boundary, a small inflow condition is set up to model a hot jet. Throughout the channel the supersonic reactive flow enters from right to left at the corresponding CJ velocity. The right boundary adopts the inflow condition and an ideal outflow condition is imposed on the left boundary, implemented by extrapolating ghost cells from the interior. 
When the moving boundary is triggered using the level-set technique, the upper expanding boundary will rotate at a constant angular velocity on the basis of the fulcrum illustrated with the circle in Fig.1. Note that, constant angles are considered in the following simulations, unless otherwise mentioned.

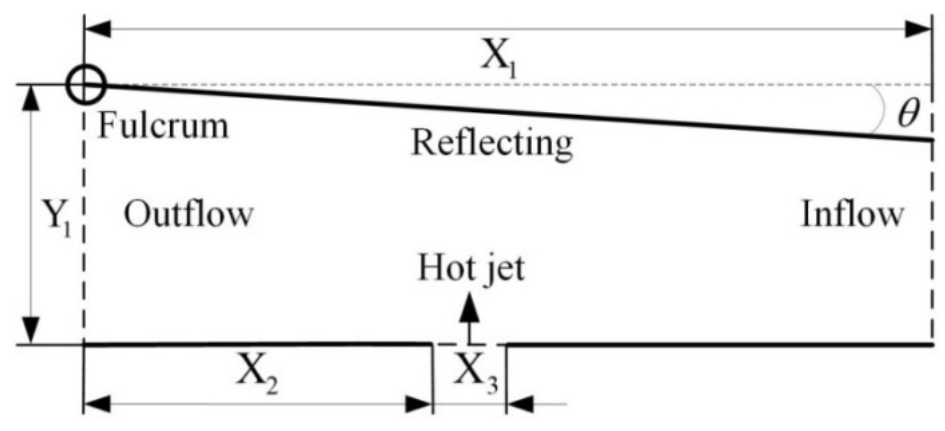

Fig.1 Schematic of the calculation model.

The inflow parameters of the hot jet are set to the values of the CJ state of a $\mathrm{H}_{2} / \mathrm{O}_{2}$ detonation under the condition of pressure $6.67 \mathrm{kPa}$ and temperature $300 \mathrm{~K}$. The injection velocity is specified as the sonic speed of the jet flow to make it a choked hot jet. When detonation initiation is realized successfully, the hot jet is switched off and the inflow condition is immediately changed to the reflecting condition. Therefore, the parameter "time" is also considered to control the injection duration of the hot jet when dealing with the boundary condition. The details of the hot jet are shown in Table 2.

Table 2 The equilibrium CJ state of the hot jet. Note that the parameters for the species are given in mass fractions.

\begin{tabular}{ccc}
\hline Parameters & Values & Unit \\
\hline Pressure & 86376 & $\mathrm{~Pa}$ \\
Temperature & 1943.8 & $\mathrm{~K}$ \\
Density & 0.155 & $\mathrm{~kg} / \mathrm{m}^{3}$ \\
Velocity & 850 & $\mathrm{~m} / \mathrm{s}$ \\
Energy & 349280 & $\mathrm{~J} / \mathrm{mol}$ \\
\hline
\end{tabular}




$\begin{array}{ll}Y_{1} & 0.0088 \\ Y_{2} & 0.9912\end{array}$

\subsection{Convergence analysis}

Three different cases of SAMR implementation are carried out for convergence analysis. In order to avoid the extra diffusion introduced by the GFM for the upper expanding wall and concentrate on the effect of grid resolution, these cases are carried out in a straight channel where the expansion angle in Fig. 1 is given $\theta=0^{\circ}$. The detailed information of the three cases is shown in Table 3.

Table 3 Mesh refinement parameters.

\begin{tabular}{cccc}
\hline & Case 1 & Case 2 & Case 3 \\
\hline \multirow{2}{*}{ Refinement } & 4 levels & 5 levels & 6 levels \\
parameters & $(2,2,2)$ & $(2,2,2,2)$ & $(2,2,2,2,2)$ \\
& $\Delta_{\text {min }}=1.56 \times 10^{-5} \mathrm{~m}$ & $\Delta_{\text {min }}=7.8 \times 10^{-6} \mathrm{~m}$ & $\Delta_{\text {min }}=3.9 \times 10^{-6} \mathrm{~m}$ \\
\hline
\end{tabular}

Fig. 2 shows the results of mesh refinement for the typical structure of the Mach stem induced by the injection of the hot jet. It is found that he overall pattern associated with the Mach stems, transverse waves, incident shocks and slip lines behind the triple points, is qualitatively similar in the three cases. These characteristic structures are all captured by the highest refinement levels, indicating that the refinement strategy established in the simulations can satisfy the requirements. As a compromise between computational cost and resolved resolution, the five-level mesh refinement of Case 2 is adopted in the following simulations.

The highest grid resolution in Case 2 of $\Delta_{\text {min }}=7.8 \times 10^{-6} \mathrm{~m}$, which corresponds to $275 \mathrm{Pts} / \mathrm{Lhr}$ (points per half reaction length), has been effectively verified in recent works [4, 10]. It is reported that the viscous scale is the smallest among the three diffusive scales (viscous shear layer, thermal 
heat conduction layer and mass diffusion layer). This indicates that a fully resolved simulation should be only limited by the viscous scale. For the highest grid resolution in Case 2, at least about 10 cells can be placed within the viscous scale, hence indicating that these diffusive scales can be fully resolved in the present simulations except for shock waves whose thicknesses are only several mean free path lengths. It should be noted that the two-dimensional high-resolution computations would represent turbulent structures not necessarily correctly as vortex stretching is omitted in two dimensions, thus pseudo-DNS is being performed.
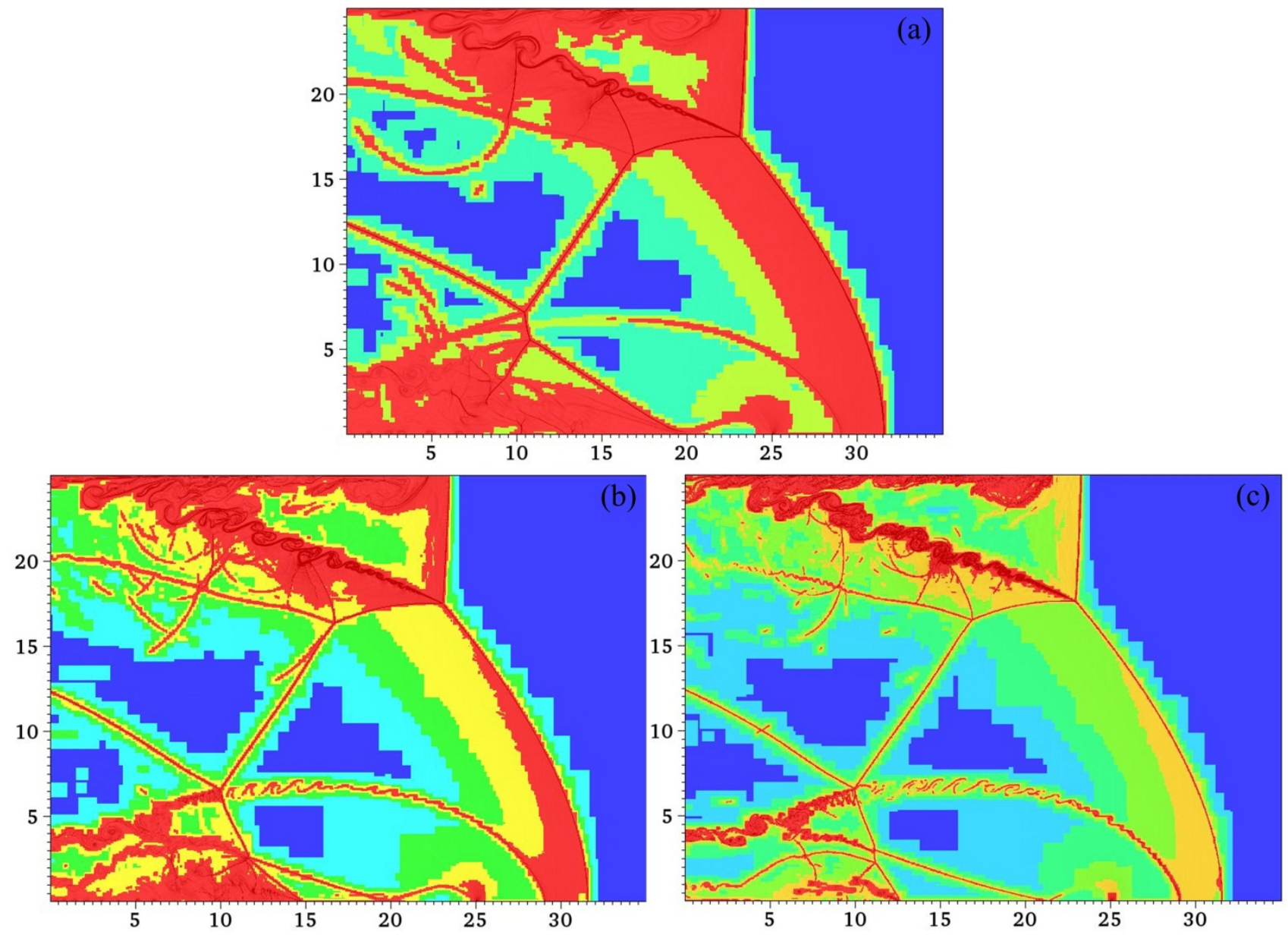

Fig.2 Three refinement cases, (a) Case 1, (b) Case 2, (c) Case 3.

\section{Stationary propagation of detonation}

The expansion angle of $\theta=3^{\circ}$ is initially employed for the expanding channel. As shown in 
Fig.3, the flow field is briefly illustrated both with and without the continuous injection of the hot jet for a quick understanding of detonation propagation in the supersonic expanding channel. In Fig.3(a) a jet is observed directly behind the Prandtl-Meyer expansion fan near the upper expanding wall. Meanwhile, near the lower wall, a triple point is going to reflect on the lower wall with detached shear layers following behind. After the triple point reflection as shown in Fig.3(b), an extended unburned jet can be observed compared with that in Fig.3(a). After the shutdown of the hot jet, the detonation wave is gradually attenuated, and the front becomes highly unstable as shown in Fig.3(c). During this period, it is observed that multiple secondary triple points are generated on the detonation front and numerous large-scale vortices are produced along the jet due to the KH (KelvinHelmholtz) instability. Subsequently, as shown in Fig.3(d) it is found that the multiple slip lines emitting from the secondary triple points are gradually converging. This indicates that the secondary triple points are getting closer to the primary one and begin the reflection on the lower wall.

Globally, it is found that the flow field in Fig.3(a) (b) is relatively laminar while it becomes highly turbulent in Fig.3(c) (d). The Reynolds number $\left(\operatorname{Re}=\frac{\rho a v t}{\mu}\right)$ is estimated using the average density, pressure and temperature at the top and bottom of the shear layer directly behind the triple point, while the time when the shear layer begins to become unstable is used. Here, the Reynolds numbers in Fig.3(a) (b) and Fig.3(c) (d) are approximated as $5 \times 10^{4}$ and $1 \times 10^{5}$, respectively. It is reported by Dimotakis [31] that $10^{5}$ is an order of magnitude larger than the typical value for the onset of turbulence on mixing layers. Therefore, it is believed that after the shutdown of the hot jet the flow field especially around the highly unstable shear layers behind the detonation front is in the turbulent regime. Different from Fig.3(a) (b), it is found that multiple pressure waves are generated along the unburned jet in Fig.3(c), which is supposed to be due to the increase in the burning rate of 
the unburned jet [32], further demonstrating the significant importance of the turbulent mixing in the consumption of the unburned jet compared with the laminar mixing.
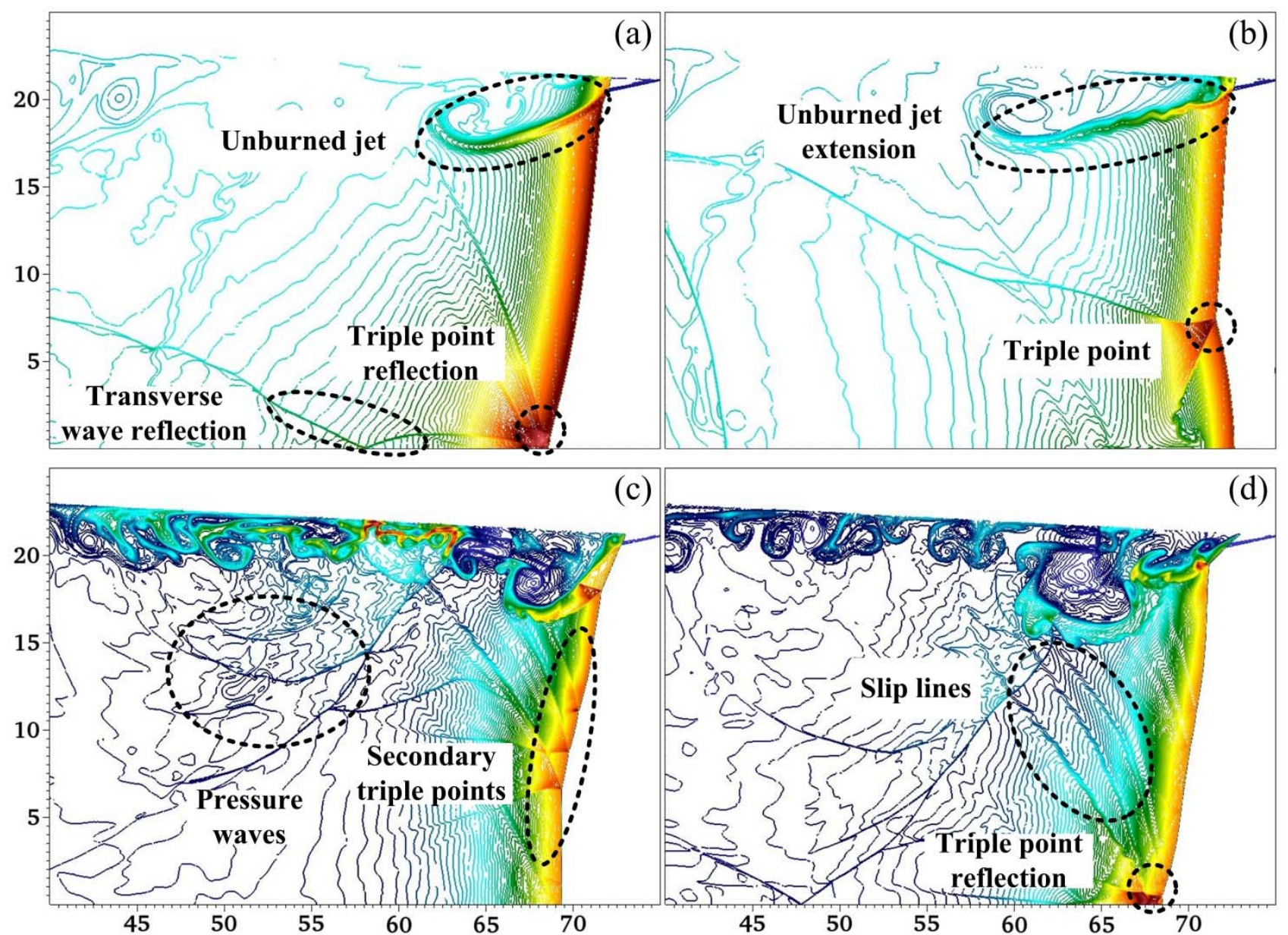

Fig.3 Density isolines showing detonation propagation in the expanding channel, with the continuous injection of the hot jet: (a) $t=470 \mu \mathrm{s}$, (b) $t=480 \mu \mathrm{s}$; after the shutdown of the hot jet: (c)

$$
t=690 \mu \mathrm{s},(\mathrm{d}) t=700 \mu \mathrm{s} .
$$

\subsection{Interactions of shear layers with unburned jet}

Fig. 4 shows the shear layer interactions with the unburned jet after the shutdown of the hot jet at $t=360 \mu \mathrm{s}$, when the initiation is fully realized in the whole supersonic flow. In addition to the primary triple point, at least four secondary ones are also produced in Fig.4(a) due to the increasing instabilities in the attenuated detonation front. The triple point is not only a source of high 
temperature and pressure due to shock compression from multiple waves, but also a source of enhanced turbulent mixing [33]. These triple points give rise to slip lines further developed into high unstable shear layers that are susceptible to the $\mathrm{KH}$ instabilities [34-35], which thus can act to enhance turbulent mixing between burned gases with unburned pockets [36-37].

Due to the formation of multiple triple points and the subsequently generated highly unstable shear layers, it is found that numerous large-scale vortices are generated underneath the upper expanding wall. Directly behind the newly generated unburned jet are partly unburned pockets left in the last period interacting with the vortices. Fig.4(b) (c) illustrate the continuous consumption of the partly unburned pockets associated with the gradual enlargement of the newly generated unburned jet. As the triple points move towards the lower wall, the Mach stem near the new unburned jet propagates forward slightly, as shown in Fig.4(b). Meanwhile, secondary triple points are swallowed by the primary one. Due to the decreased triple points and the subsequently generated highly unstable shear layers, the shear layer interactions with the unburned jet are suppressed slightly, and hence fewer vortices are produced along the unburned jet. At the same time, the partly unburned pockets are further mixed with burned gases through the engulfment of the vortices. These interactions between the shear layers emitting from triple points and partly unburned pockets, can effectively enhance the mixing of the unburned pockets with neighboring burned gases, which can facilitate the consumption of the unburned pockets through the enhanced diffusion effect. It is observed that numerous vortices involved in the partly unburned pockets become further enlarged while the mass fractions of the unburned pockets are gradually decreased. As a result, the left partly unburned pockets are basically completely consumed in Fig.4(c). Different from Fig.4(b), a new primary triple point is generated underneath the new unburned jet in Fig.3(c). When the newly generated triple point moves towards the lower wall, a secondary one is produced between the two 
primary ones, as shown in Fig.4(d). These newly generated primary and secondary triple points contribute significantly to the turbulent mixing between burned gases and unburned pockets, hence enhancing the subsequent consumption of the unburned pockets.

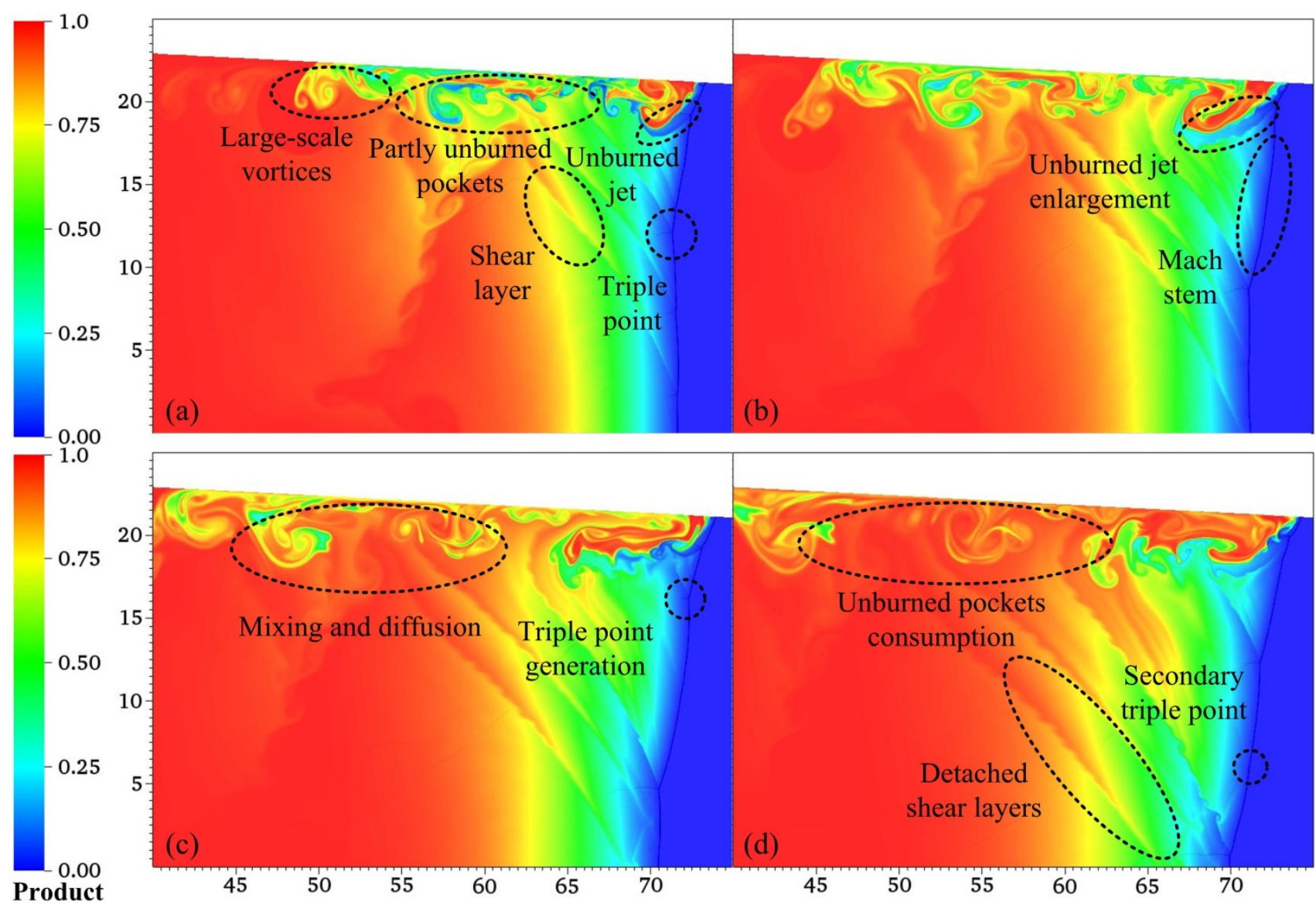

Fig.4 Contours of product mass fractions layered with density schlieren images illustrating shear

layer interactions with the unburned jet, (a) $t=565 \mu \mathrm{s}$, (b) $t=570 \mu \mathrm{s}$, (c) $t=575 \mu \mathrm{s}$, (d)

$$
t=580 \mu \mathrm{s} .
$$

For the weakly unstable detonation utilized in the present simulations, basically the reactant could be effectively consumed through adiabatic compression of the detonation wave. However, in the expanding channel due to the Prandtl-Meyer expansion fan an unburned jet is generated behind the detonation front. The consumption of the unburned jet cannot be fully completed through adiabatic compression but involves rapid turbulent mixing and diffusion through the interactions with the shear layers. It should be noted that the transverse wave interactions with the unburned jet 
can also accelerate the generation of large-scale vortices due to the RM (Richtmyer-Meshkov) instability, thus enhancing the diffusion and mixing effects. However, the transverse waves are relatively weak in weakly unstable detonations [32]. Rather than the transverse waves, highly unstable shear layers emitting from multiple triple points contribute significantly to the consumption of numerous unburned pockets through the rapid turbulent mixing and diffusion, globally playing a dominant role in the general shear layer interactions with the unburned pockets.

\subsection{Stationary propagation}

In order to conduct a direct comparison, Fig.5 illustrates the trajectories of the detonation front both with and without the hot jet injection, where the coordinate in the $X$-direction of the detonation front at $Y=12.5 \mathrm{~mm}$ is recorded in real time. As shown in Fig.5(a), when the hot jet is injected continuously the average period of the detonation oscillation is estimated as $T_{\text {con }}=50.6 \mu \mathrm{s}$. Within a full period, two secondary oscillations are also observed, with the corresponding periods of $T_{1}=28.69 \mu \mathrm{s}$ and $T_{2}=21.9 \mu \mathrm{s}$, respectively. The two secondary ones differ little from each other due to the nonuniformity resulting from the Prandtl-Meyer expansion. Even though there exist periodic oscillations, the curve on the whole presents a straight trend, demonstrating that the detonation propagates forward at a constant relative velocity of $v=181.63 \mathrm{~m} / \mathrm{s}$ represented by the constant slope of the curve. Therefore, the absolute velocity of the detonation is calculated as $V=$ $v+V_{C J}=1769.47 \mathrm{~m} / \mathrm{s}$, and the subsequent overdrive degree is evaluated as $f=1.242\left(f=\left(V / V_{C J}\right)^{2}\right)$, indicating that a slightly overdriven detonation is formed even with the expansion fan in the expanding channel when the hot jet is injected continuously.

When the hot jet is shut down at $t=360 \mu \mathrm{s}$, the slope of the trajectory curve is gradually decreasing as shown in Fig.5(b), demonstrating that the detonation is gradually attenuating. 
Eventually at approximately $t=500 \mu$ s, the slope of the curve decreases to zero accompanied by periodic oscillations at the position of $X=71 \mathrm{~mm}$, suggesting the formation of a dynamically stationary detonation in the expanding channel. The oscillation period is estimated as about $T_{\text {sh }}=57.5 \mu \mathrm{s}$, which is $13.6 \%$ larger than that with the continuous injection of the hot jet due to the decreased strength of the attenuated detonation. It should be noted that, within the primary period in Fig.5(b), secondary oscillations are also generated but are more irregular than that in Fig.5(a), which might result from the multiple secondary triple points in the detonation front after the shutdown of the hot jet, speculating that the degree of secondary oscillation irregularity can represent the irregularity of generation and evolution of secondary triple points.
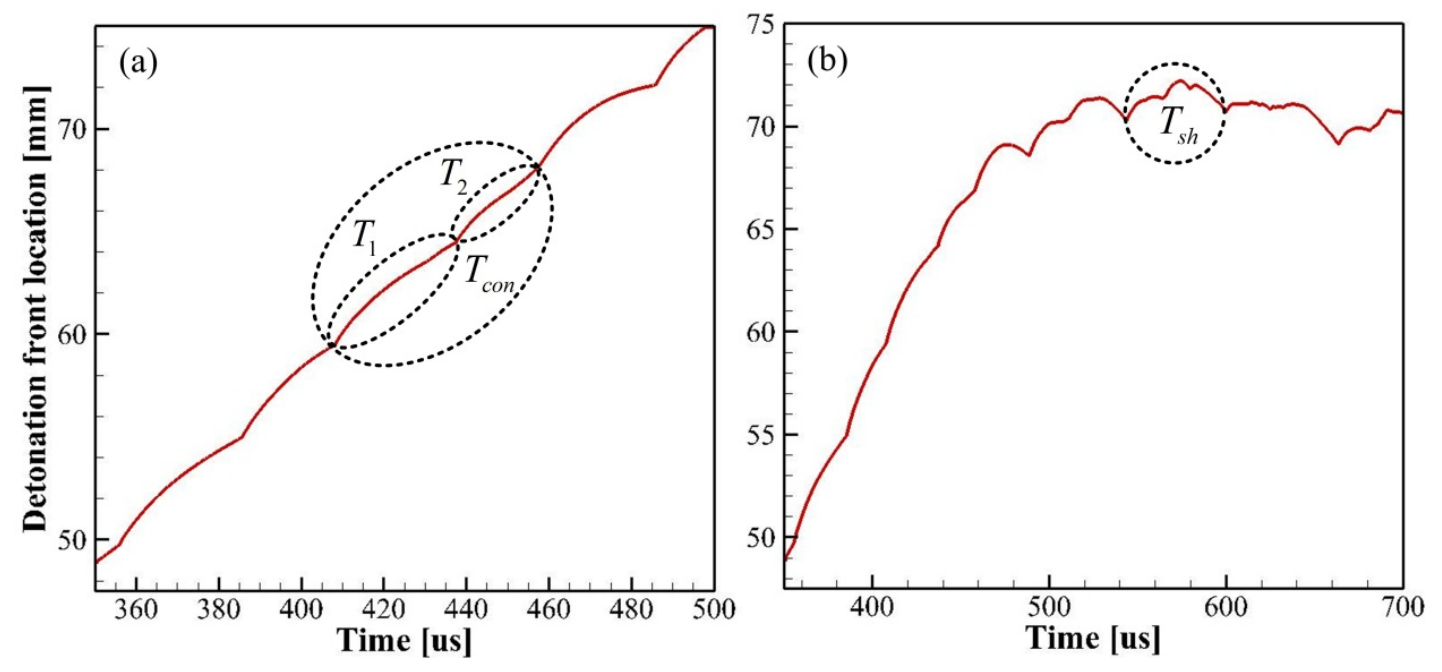

Fig. 5 The trajectories of the detonation front, (a) with the continuous hot jet injection, (b) after the hot jet shutdown.

\subsection{Stabilization mechanism}

It has been demonstrated that in straight channels overdriven detonation can attenuate to CJ detonation and dynamically stable propagation of detonation can be achieved when the supersonic incoming flow is given the corresponding CJ velocity after the shutdown of the hot jet [4]. Different from straight channels, the product in expanding channels is expected to expand more freely due to 
the Prandtl-Meyer expansion, which is theoretically able to result in further attenuation of CJ detonation and even detonation failure. However, rather than further attenuation or detonation failure, dynamic detonation stabilization is realized in the expanding channel after the shutdown of the hot jet.

As discussed above, in the expanding channel the interactions between the shear layers and the unburned jet (also including numerous unburned pockets separated from the unburned jet) enhance the turbulent mixing, thus facilitating the consumption of the unburned jet. The subsequent accelerated heat release through the rapid consumption of the unburned jet to some degree can counteract the additional attenuation effect resulting from the expansion wave in the expanding channel, thus preventing the detonation from further attenuation.

Meanwhile, highly unstable shear layers along the unburned jet can be considered as free boundaries, together making up of a hydrodynamic channel with the lower wall, as shown in Fig.6. It should be noted that the shear layer is a free boundary only for the fluid, but not for the acoustic waves. The latter can still penetrate the shear layer and thus influence the pressure field. Within the channel a dynamic hydrodynamic "throat" can be identified where the Mach number is unity. The hydrodynamic "throat" can separate the dynamic stationary detonation structure from the downstream unsteady flow, as is sketched in Fig.6(a). This is also supported by the identification of an effective sonic surface in Fig.6(b), which separates the stationary detonation structure from the unsteady flow. The sonic lines in Fig.6(b) are obtained by first calculating the global Mach number for the flow field and then exactly figuring out the lines where the Mach number equals to unity. 

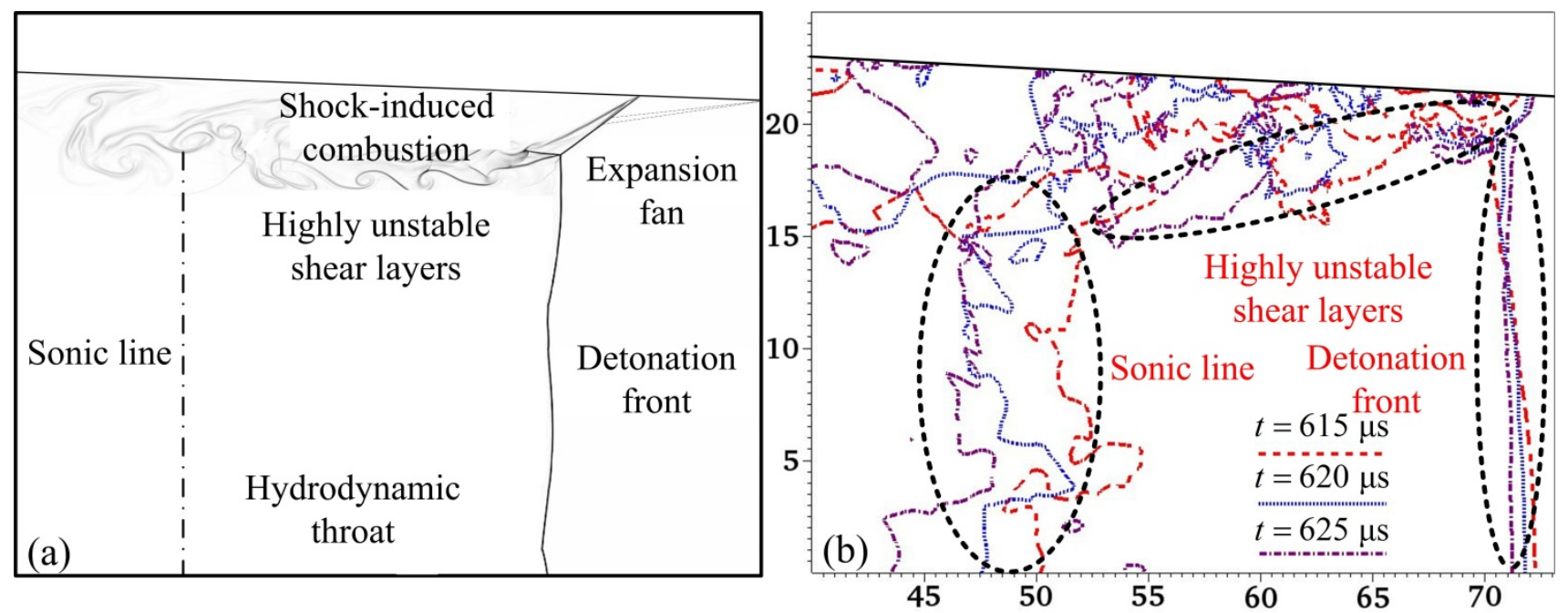

Fig.6 The hydrodynamic channel, (a) schematic of the hydrodynamic channel and (b) sonic lines sketching the hydrodynamic channel.

The formation of the hydrodynamic channel in Fig.6(a) can suppress the expansion of the detonation product, hence increasing the pressure behind the detonation front and eventually enhancing the detonation strength which can prevent further detonation attenuation effectively. As a result, dynamically stationary propagation of detonation is achieved eventually with the help of the hydrodynamic channel. Dynamic evolution of the hydrodynamic channel using unstable sonic lines is illustrated in Fig.6(b) with three successive frames. Since the supersonic incoming velocity is given the corresponding $V_{C J}$, the detonation can be regarded as a $\mathrm{CJ}$ one when it realizes dynamically stationary propagation. It is reported that unburned pockets, burned inside within the reaction zone in front of the sonic line, are separated from the downstream rarefaction by a surface similar to what would be the CJ point in the case of a one-dimensional steady-state detonation [38]. Similarly, the sonic lines in Fig.6(b) present a rough idea of the position of the analog CJ point, which is sketched by the hydrodynamic "throat". It can be found that the global structure sketched by the sonic lines in Fig.6(b), is very close to the hydrodynamic "channel" in Fig.6(a), indicating that the hydrodynamic "channel" makes sense. 
Therefore, the mechanism of dynamically stationary propagation of detonation in supersonic expanding channels can be summarized as follows: first, due to the rapid turbulent mixing and diffusion effects resulting from interactions between the shear layers and the unburned jet, the generation and consumption of the unburned jet are accelerated for rapid periodic heat release, thus supporting the stationary propagation of detonation; second, due to the formation of the hydrodynamic "channel" associated with the hydrodynamic "throat", further detonation attenuation is effectively suppressed. The combined two effects eventually lead to the formation of dynamically stationary propagation in supersonic expanding channels.

It should be noted that in the present study the height of the expanding channel is given the size of the detonation cell. If the channel height varies, the detonation stability may also change. Based on the mechanism discussed above, it is known that dynamic propagation of detonation in supersonic expanding channels is mainly determined by the unburned jet and the hydrodynamic channel. The generated unburned jet mainly depends on the expansion angle and the inflow; however, the hydrodynamic "channel" might be significantly influenced by the height of the expanding channel. When the height of the expanding channel is large, the hydrodynamic "channel" can occupy a large part of the whole channel, thus contributing significantly to the detonation stability in the supersonic flow. On the contrary, if the height of the expanding channel is small, the hydrodynamic "channel" only occupies a relatively small part of the whole channel, hence not benefiting the dynamic detonation stabilization.

\subsection{Application of stabilization control}

Four different supersonic expanding channels with the corresponding expansion angles $\left(4^{\circ}, 6^{\circ}\right.$, $8^{\circ}, 10^{\circ}$ ) are utilized as shown in Fig.7. When the expansion angle is $4^{\circ}$, after the shutdown of the hot 
jet at $t=360 \mu$ s the unburned jet is relatively small and almost all reacted in front of the sonic line, as shown in Fig.7(a). However, with the increase of the expansion angle, the unburned jet gradually becomes larger and is even extended out of the outflow boundary. When the expansion angle is $6^{\circ}$, only a partially unburned pocket is left behind the sonic line, as shown in Fig.7(b); however, when the expansion angles are increased to $8^{\circ}$ and $10^{\circ}$ in Fig.7(c)(d) respectively, numerous unburned pockets are produced along the unburned jet due to the KH instability. Especially in Fig.7(d), it can be observed that the majority of the unburned jet is not reacted in front of the sonic line.

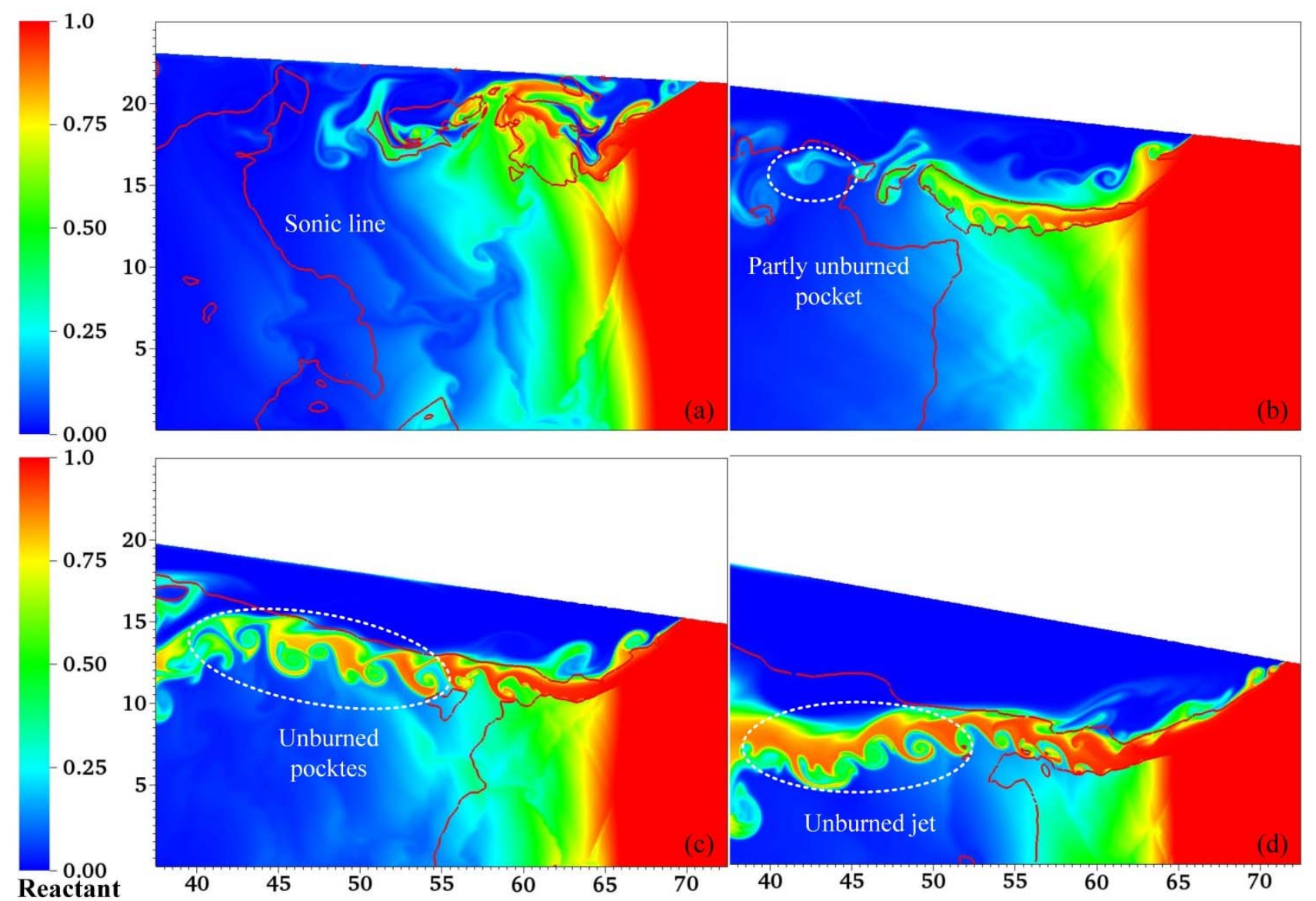

Fig.7 Contours of reactant mass fractions layered with sonic lines illustrating detonation propagation for four expansion angles, (a) expansion angle of $4^{\circ}$, (b) expansion angle of $6^{\circ}$, (c) expansion angle of $8^{\circ},(d)$ expansion angle of $10^{\circ}$

As the expansion angle increases, the enlarged unburned jet near the upper wall gradually leads to the formation of more large-scale vortices along the jet boundary due to hydrodynamic 
instabilities. This can make the flow field more turbulent on the one hand and thus enhance the mixing and diffusion effects to facilitate the consumption of the unburned jet and the subsequent heat release; however, on the other hand the consumption of the enlarged unburned jet becomes more time expensive at the same time. The existence of the enlarged unburned jet that gets swept downstream results in an increase in the effective length of the reaction zone of the detonation wave. With the increase of the expansion angle, more delayed heat release from these unburned pockets, separated from the enlarged unburned jet left behind the sonic line, cannot contribute to dynamically stationary propagation of detonation, which eventually leads to easier detonation failure. It is suggested that when the expansion angle is relatively small, the heat release from the consumption of the unburned jet facilitated by the mixing and diffusion effects can effectively support the detonation stabilization. However, when the expansion angle is relatively large, the dynamic stabilization of detonation cannot be maintained due to the delayed consumption of the enlarged unburned jet. Therefore, it is reasonably supposed that there might exist some critical angle $\theta_{\mathrm{CT}}$. When the angle is smaller than $\theta_{\mathrm{CT}}$, dynamic stabilization of detonation can be realized in expanding channels while the detonations propagate below the $V_{C J}$ and finally fails when the angle is larger than $\theta_{\mathrm{CT}}$. This critical value $\theta_{\text {CT }}$ is related with the incoming Mach number (which has a significant influence on the generation of the unburned jet resulting from the expansion fan), the height of the channel (which determines the role of the unburned jet on the whole detonation propagation), and even the mixture characteristics (which define the diffusion and mixing performance), the latter aspect still needing further investigations.

Even though the unburned jet is almost consumed in front of the sonic line when the expansion angle is relatively small, the increased fluctuations of shear layers resulting from large-scale vortices and pressure oscillations mainly due to the increase in the burning rate of the unburned jet [32] can 
still weaken the stability of the hydrodynamic channel, thus eventually leading to detonation attenuation and further failure. As indicated by the curve of "Fixed boundary" in Fig.8 with the expansion angle of $4^{\circ}$, the detonation wave continues its advected backward propagation and will eventually propagate out of the channel, hence resulting in the failure of dynamic stabilization in the supersonic expanding channel.

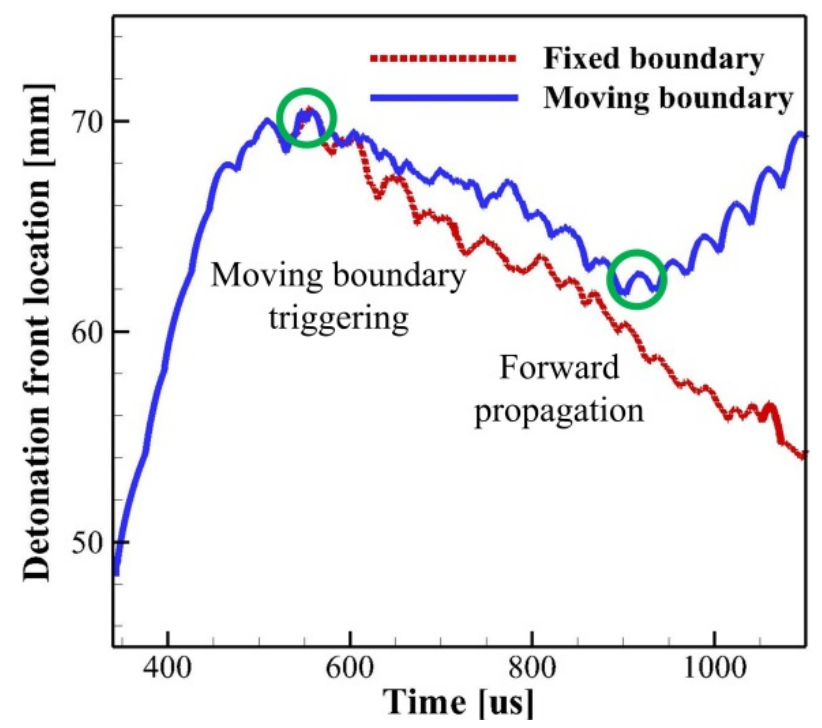

Fig.8 The trajectories of the detonation front using both the fixed and moving boundaries.

Based on the preceding mechanism, the control application is conducted to achieve dynamic stabilization using a moving boundary technique. This can change the expansion angle to further adjust the hydrodynamic channel dynamically and have a significant influence on the shear layer interaction with the unburned jet. The upper expanding boundary is gradually lifting up at a constant angular velocity of $\frac{\pi}{0.00036 \times 45}$ on the basis of the fulcrum. Before the triggering of the moving boundary, the backward velocity during detonation attenuation is approximately $30.5 \mathrm{~m} / \mathrm{s}$, whereas after the triggering of the moving boundary at $60 \%$ the backward velocity is decreased to $18.2 \mathrm{~m} / \mathrm{s}$ as indicated by the curve of "Moving boundary", which is about $60 \%$ of the backward velocity before the triggering. It should be noted that, at about $T=900 \mu$ s the detonation turns to forward propagation successfully from backward propagation, indicating that further attenuation or even 
failure is prevented effectively. Furthermore, when the forward propagation has continued for a while, a new triggering of the moving boundary can be set to gradually increase the expansion angle on the basis of the fulcrum. This is supposed to change the forward moving detonation to detonation attenuation once again, thus being able to dynamically maintain the detonation with a limited length in the expanding channel. Therefore, through the dynamic control of moving boundary, dynamically stationary propagation of detonation can be realized in supersonic expanding channels eventually. It should be noted that the moving boundary adjustment is not restricted to a change between 4 and 3 degrees. The angular velocity and moving time can be adjusted based on the initially given expansion angle. As indicated in Fig.7, detonation failure does not occur immediately even for large expansion angles, but will first undergo a gradual attenuation. Therefore, when given a proper angular velocity and moving time, dynamic detonation stabilization can still be realized even for larger angles using the moving boundary before the eventual extinction.

\section{Discussion}

\subsection{Three-dimensional effect}

In the present work two-dimensional pseudo-DNS are carried out solving the NS equations with the SAMR framework. It is reported that the turbulence in detonations is mostly generated by the RM instability through the baroclinic vorticity generation mechanism [39]. This can lead to vorticity generation comparable to the system scale. Both theoretical analysis and high-resolution numerical simulations were utilized to compare two-dimensional and three-dimensional growth rates of the RM instability [40]. It was found that the three-dimensional growth rate in the nonlinear regime is about 
$20 \%$ larger and $25 \%$ faster than that in two-dimensional simulations. However, in the linear regime the growth rates of the instability were the same in two and three dimensions. Oran and Gamezo [37] carried out investigations of flame acceleration and DDT, and showed that two-dimensional computations agree well with three-dimensional results and experimental observations. Even though the accelerating flame became more wrinkled in three-dimensional simulations, the overall flame development is dominated by the RM instability [36], concluding that the two-dimensional and three-dimensional simulations showed very similar results. In the present study, the RM instability tends to be a dominant mechanism generating turbulent structures around the unburned jet and behind the detonation front. Although the two-dimensional computations qualitatively agree with three-dimensional simulations, in order to clarify the exact detonation evolution in supersonic flows for the further application, three-dimensional computations should be carried out in the future.

\subsection{Effect of one-step chemistry}

A single-step chemical kinetic has been adopted in the present work. It is reported that one of the failures of the one-step model is its inability to capture the chain-branching character of the reactions and the corresponding cross-over effect [41]. Nonetheless, there are additional issues that make the simulation of gaseous detonations challenging, including the three-dimensional nature of the coherent structures and turbulent flow in the reaction zone, the challenge of carrying out highorder simulations needed for turbulence modelling, the storage requirements for detailed chemical reaction mechanisms [42], and so on. Hence, most detonation simulations have been carried out using a simplified chemical kinetic model in multiple dimensional detonation simulations [25-27]. Even with the simple reaction model, the results are in qualitative agreement with the experimental observations [45]. A small number of simulations have been carried out with detailed chemical 
reaction mechanisms for hydrogen-oxygen mixtures [46-47]. However, the detonation structures obtained using detailed kinetics for stable detonations are very similar to those with the Arrhenius kinetics [48]. Instead, it is more important to have an accurate model of the turbulent diffusive flow field coupled to the single step reaction model for the chemical energy release. After developing a proper fluid dynamics turbulent diffusive model, using realistic detailed kinetics is useful for more accurate detonation simulations.

\section{Conclusions}

In the present work, dynamic detonation stabilization of supersonic combustible mixtures in expanding channels, including its mechanism and control application, is investigated solving the reactive NS equations and one-step two-species reaction model using the hybrid sixth-order WENOCD scheme based on the SAMR framework. The main results are as follows:

(1) The numerous large-scale vortices, mainly resulting from the shear layer interactions with the unburned jet, play a significant role in the rapid turbulent mixing and diffusion effects, which contribute significantly to the consumption of the unburned jet and its subsequent heat release and hence support dynamically stationary propagation of detonation.

(2) In addition to the shear layer interactions with the unburned jet, due to the formation of the hydrodynamic channel associated with a hydrodynamic throat, detonation attenuation is effectively suppressed. The combined two effects resulting from both the shear layer interactions with the unburned jet and the hydrodynamic channel eventually lead to dynamically stationary propagation in expanding channels.

(3) With the increase of the expansion angle, the unburned jet gradually becomes enlarged, thus taking more time for the consumption. When the unburned jet is even extended out of the 
sonic line in the hydrodynamic channel, the deficit of heat release cannot contribute to stationary propagation, and eventually leads to detonation failure.

(4) There might exist a critical angle $\theta_{\text {Ст. Dynamic stabilization of detonation can be realized }}$ in expanding channels when the angle is smaller than $\theta_{\mathrm{CT}}$, while the detonation propagates below $V_{C J}$ and finally fails when the angle is larger than $\theta_{\mathrm{CT}}$.

(5) Through the dynamic control of moving boundary, continuous detonation attenuation is suppressed to turn to forward propagation successfully, indicating that dynamically stationary propagation of detonation can be effectively realized through the dynamic control of a moving boundary.

\section{Acknowledgements}

This work is supported by National Natural Science Foundation of China (No. 11702323), National Foundation of Defense Technology (No. 3101032) and Postdoctoral Innovative Talent Support Program (No. BX20180372).

\section{References}

[1] K. Kailasanath, Review of propulsion applications of detonation waves. AIAA J. 38 (2000) 1698-1708.

[2] P. Wolański, Detonative propulsion. Proc. Combust. Inst. 34 (2013) 125-158.

[3] X.D. Cai, J.H. Liang, R. Deiterding, Y. Mahmoudi, M.B. Sun, Experimental and numerical investigations on propagating modes of detonations: Detonation wave/boundary layer interaction. Combust. Flame 190 (2018) 201-215.

[4] X.D. Cai, R. Deiterding, J.H. Liang, M.B. Sun, Y. Mahmoudi, Diffusion and mixing effects in 
hot jet initiation and propagation of hydrogen detonations J. Fluid Mech. 836 (2018) 324-351.

[5] X.D. Cai, J.H. Liang, R. Deiterding, Z.Y. Lin, Detonation simulations in supersonic combustible mixtures with nonuniform species. AIAA J. 54 (2016) 2449-2462.

[6] X.D. Cai, J.H. Liang, R. Deiterding, Z.Y. Lin, M.B. Sun, Detonation interaction with cavity in supersonic combustible mixture. AIAA J. 56 (2018) 2096-2102.

[7] H.W. Yeom, B.G. Seo, H. G. Sung, Numerical analysis of a scramjet engine with intake sidewalls and cavity flameholder. AIAA J. 51 (2013) 1566-1575.

[8] B.J. Colcord, W.A. Sirignano, F. Liu, Flameholding in converging and turning channels over cavities with periodic port injection. AIAA J. 51 (2013) 1621-1630.

[9] J.W. Kirik, C.P. Goyne, S.J. Peltier, C.D. Carter, M.A. Hagenmaier, Velocimetry measurements of a scramjet cavity flameholder with inlet distortion. J. Propul. Power, 30 (2014) 1568-1576.

[10] X.D. Cai, R. Deiterding, J.H. Liang, Y. Mahmoudi, Adaptive simulations of viscous detonations initiated by a hot jet using a high-order hybrid WENO-CD scheme. Proc. Combust. Inst. 36 (2017) 2725-2733.

[11] M.I. Radulescu, G.J. Sharpe, C.K. Law, J.H.S. Lee, The hydrodynamic structure of unstable cellular detonations. J. Fluid Mech. 580 (2007) 31-81.

[12] K. Mazaheri, Y. Mahmoudi, M.I. Radulescu, Diffusion and hydrodynamic instabilities in gaseous detonations. Combust. Flame 159(6) (2012) 2138-2154.

[13] K. Mazaheri, Y. Mahmoudi, M. Sabzpooshani, M.I. Radulescu, Experimental and numerical investigation of propagation mechanism of gaseous detonations in channels with porous walls. Combust. Flame 162 (2015) 2638-2659.

[14] F.K. Lu, E.M. Braun, Rotating detonation wave propulsion: Experimental challenges, 
modeling, and engine concepts. J. Propul. Power 30(5) (2014) 1125-1142.

[15] S.P.M. Bane, J.L. Ziegler, J.E. Shepherd, Development of One-Step Chemistry Models for Flame and Ignition Simulation, Report No. GALCITFM: 2010.002, California Institute of Technology, 2010.

[16] D.J. Hill, D.I. Pullin, Hybrid tuned center-difference-WENO method for large eddy simulations in the presence of strong shocks. J. Comput. Phys. 194 (2004) 435-450.

[17] C. Pantano, R. Deiterding, D.J. Hill, D.I. Pullin. A low numerical dissipation patch-based adaptive mesh refinement method for large-eddy simulation of compressible flows. J. Comput. Phys. 221 (2007) 63-87.

[18] R. Deiterding, Parallel adaptive simulation of multi-dimensional detonation structures, $\mathrm{PhD}$ thesis, Technical University of Cottbus, Cottbus, Germany, 2003.

[19] Z. Liang, S. Browne, R. Deiterding, J.E. Shepherd, Detonation front structure and the competition for radicals Proc. Combust. Inst. 31(2) (2007) 2445-2453.

[20] J.L. Ziegler, R. Deiterding, J.E. Shepherd, D.I. Pullin, Simulations of compressible, diffusive, reactive flows with detailed chemistry using a high-order hybrid WENO-CD scheme. J. Comput. Phys. 230(20) (2011) 7598-7630.

[21] K.P. Grogan, M. Ihme, Weak and strong ignition of hydrogen/oxygen mixtures in shock tube systems. Proc. Combust. Inst. 35 (2015) 2181-2189.

[22] M.I. Radulescu, G.J. Sharpe, J.H.S. Lee, C.B. Kiyanda, A.J. Higgins, R.K. Hanson, The ignition mechanism in irregular structure gaseous detonations. Proc. Combust. Inst. 30 (2005) 1859-1867.

[23] M.I. Radulescu, A detonation paradox: Why inviscid detonation simulations predict the incorrect trend for the role of instability in gaseous cellular detonations. Combust. Flame 195 
(2018) 151-162.

[24] G.B. Goodwin, R.W. Houim, E.S. Oran, Shock transition to detonation in channels with obstacles. Proc. Combust. Inst. 36 (2017) 2717-2724.

[25] H.H. Teng, H.D. Ng, K. Li, C.T. Luo, Z.L. Jiang, Evolution of cellular structures on oblique detonation surfaces. Combust. Flame 162 (2015) 470-477.

[26] X.D. Cai, J. H. Liang, Z.Y. Lin, R. Deiterding, H. Qin, and X. Han, Adaptive mesh refinement based numerical simulation of detonation initiation in supersonic combustible mixtures using a hot jet, ASCE J Aerosp. Eng. 28 (2015) 04014046.

[27] X.C. Mi, A.J. Higgins, H.D. Ng, C.B. Kiyanda, and N. Nikiforakis, Propagation of gaseous detonation waves in a spatially inhomogeneous reactive medium, Phys. Rev. Fluids 2 (2017), 053201.

[28] M. Lombardini, Richtmyer-Meshkov instability in converging geometries, $\mathrm{PhD}$ thesis, California Institute of Technology, California, USA, 2008.

[29] P. Kaps, P. Rentrop, Generalized Runge-Kutta methods of order four with step size control for stiff ordinary differential equations. Numer. Math. 33 (1979) 55-68.

[30] R. Deiterding, A parallel adaptive method for simulating shock-induced combustion with detailed chemical kinetics in complex domains. Comput. Struct. 87 (2009) 769-783.

[31] P.E. Dimotakis, Turbulent mixing. Annu. Rev. Fluid Mech. 37 (2005) 329-356.

[32] J.H.S. Lee, The Detonation Phenomena, Cambridge University Press, Cambridge, New York, 2008.

[33] B.M. Maxwell, R.R. Bhattacharjee, S.S.M. Lau-Chapdelaine, S.A.E.G. Falle, G.J. Sharpe, M.I. Radulescu, Influence of turbulent fluctuations on detonation propagation. J. Fluid Mech. 818 (2017) 646-696. 
[34] L. Massa, J.M. Austin, T.L. Jackson, Triple-point shear layers in gaseous detonation waves. J. Fluid Mech. 586 (2007) 205-248.

[35] R.R. Bhattacharjee, S.S.M. Lau-Chapdelaine, G. Maines, L. Maley, M.I. Radulescu, Detonation re-initiation mechanism following the Mach reflection of a quenched detonation. Proc. Combust. Inst. 34 (2013) 1893-1901.

[36] V.N. Gamezo, T. Ogawab, E.S. Oran, Numerical simulations of flame propagation and DDT in obstructed channels filled with hydrogen-air mixture. Proc. Combust. Inst. 31(2) (2007) 24632471.

[37] E.S. Oran, V.N. Gamezo, Origins of the deflagration-to-detonation transition in gas-phase combustion. Combust. Flame 148 (2007) 4-47.

[38] V.N. Gamezo, D. Desbordes, E.S. Oran. Two-dimensional reactive flow dynamics in cellular detonation waves. Shock Waves 9 (1999) 11-17.

[39] M. Brouillette, The Richtmyer-Meshkov instability, Annu. Rev. Fluid Mech. 34 (2002) 445 468 .

[40] X.L. Li, Q. Zhang, A comparative numerical study of the Richtmyer-Meshkov instability with nonlinear analysis in two and three dimensions, Phys. Fluids 9 (1997) 3069-3077.

[41] B. Varatharajan, F.A. Williams, Chemical-kinetic descriptions of high-temperature ignition and detonation of acetylene--oxygen-diluent systems. Combust. Flame 124 (2001), 624-645.

[42] J. E. Shepherd, Detonation in gases. Proc. Combust. Inst. 32 (2009), 83-98.

[43] M.I. Radulescu, B. MCN. Maxwell, The mechanism of detonation attenuation by a porous medium and its subsequent re-initiation. J. Fluid Mech. 667 (2011), 96-134.

[44] X.D. Cai, J. H. Liang, R. Deiterding, Numerical investigation on detonation control using a pulse hot jet in supersonic combustible mixture. Combust. Sci. Technol. 188 (2016), 1674- 
1690.

[45] Y. Wang, W. Han, R. Deiterding, Z. Chen, Effects of disturbance on detonation initiation in $\mathrm{H}_{2}-\mathrm{O}_{2}-\mathrm{N}_{2}$ mixture. Phys. Rev. Fluids 3 (2018), 123201.

[46] G.J. Sharpe, Transverse wave in numerical simulations of cellular detonation. J. Fluid Mech. 447 (2011), 31-51. 